\title{
Análise volumétrica do pólen de Attalea funi- fera Mart. (Arecaceae) em amostras de pólen apícola do litoral do baixo sul da Bahia, Brasil
}

Volumetric analysis of Attalea funifera Mart. (Arecaceae) pollen in bee pollen samples from the low southern coast of Bahia, Brazil

Rodolfo F. de Alves $1^{\oplus 凶}$, Francisco de Assis R. dos Santos $1^{\oplus}$

\begin{abstract}
1. Departamento de Ciências Biológicas, Universidade Estadual de Feira de Santana, Feira de Santana, Bahia, Brasil
\end{abstract}

\section{Palavras-chave:}

Apicultura. Pólen apícola. Palinologia. Volume polínico. Apis mellifera .

\section{Keywords:}

Beekeeping. Bee pollen. Palynology. Pollen volume. Apis mellifera.

Recebido em: 04/12/2018

Aceito em: 20/12/2018

\section{Resumo}

Foi realizada a análise volumétrica do pólen de Attalea funifera Mart. (Arecaceae) em amostras de pólen apícola comercializadas no município de Nilo Peçanha, Bahia, no período de maio a novembro de 2014. As amostras foram identificadas e quantificadas, no intuito de determinar a origem botânica e a importância de A. funifera na composição do pólen apícola, sob o aspecto volumétrico. O espectro polínico foi composto por 23 tipos polínicos distribuídos em 13 famílias botânicas, tendo como as mais representativas as famílias Fabaceae, Euphorbiaceae e Asteraceae. O tipo polínico mais frequente foi Mimosa pudica, porém, as análises volumétricas apontaram A. funifera como o mais importante na constituição do pólen coletado. Portanto, com base no volume $\left(93.974,70 \mu \mathrm{m}^{3}\right)$, verifica-se o potencial do pólen de $A$. funifera em amostras de pólen apícola e, consequentemente, a importância de análises volumétricas em adição às análises quantitativas e de frequência.

\begin{abstract}
The volumetric analysis of Attalea funifera Mart. (Arecaceae) pollen was carried out. Commercialized bee pollen samples were collected from May to November 2014 in Nilo Peçanha, Babia State. The pollen samples were identified and quantified in order to determine their botanical origin and to analyze the importance of $A$. funifera to bee pollen composition, under the volumetric aspect. The pollen spectrum was composed of 23 pollen types distributed in 13 botanical families, especially Fabaceae, Euphorbiaceae and Asteraceae. The most frequent pollen type was Mimosa pudica, but the volumetric analyzes indicated $A$. funifera as the most important in the constitution of the pollen collected. Therefore, based on the volume $\left(93,974.70 \mu^{3}\right)$, the potential of A. funifera pollen is verified in bee pollen samples and, consequently, the importance of volumetric analysis in addition to quantitative and frequency analyzes.
\end{abstract}

\section{Introdução}

O pólen apícola é um dos produtos encontrados dentro de uma colmeia, obtido pela agregação de diferentes grãos de pólen coletados pelas abelhas. Segundo a normativa n. ${ }^{\circ} 3$, de 19 de janeiro de 2001, do Ministério de Agricultura e do Abastecimento, esse produto é definido como o resultado da aglutinação do pólen das flores, efetuada pelas abelhas operárias, mediante a adição de néctar e de substâncias salivares (Brasil, 2001).

No Brasil, a produção de pólen apícola foi iniciada modestamente no final da década de 1980. Nos dias atuais, o mercado é favorável ao consumo de produtos naturais, complementares à dieta ou com efeitos terapêuticos e, com isso, há o estímulo e a promoção do desenvolvimento da cadeia apícola (Barreto et al., 2005). Segundo dados do IBGE, a região que 
mais se destaca na produção do pólen apícola é o Nordeste, reconhecido como uma das áreas de maior potencial para a apicultura no país, principalmente os estados do Piauí, Ceará e Bahia. Entre os municípios baianos, a região do baixo sul apresenta um grande potencial para o desenvolvimento dessa atividade, principalmente para o pólen apícola monofloral, por conta das vastas plantações de palmeira, especialmente de dendê (Elaeis guineensis L.) e piaçava (Attalea funifera Mart.).

As palmeiras (Arecaceae) são representadas atualmente por 181 gêneros e, aproximadamente, 2.600 espécies. No Brasil, ocorrem 37 gêneros e cerca de 299 espécies (Flora do Brasil, 2018). São plantas com grande potencial na produção de pólen apícola monofloral (Alves; Santos, 2017). Entre as espécies que ocorrem na Bahia, A. funifera é endêmica do sul do estado, com predomínio na zona costeira, principalmente nos municípios de Cairu, Ilhéus, Nilo Peçanha, Taperoá e Valença (Avelar, 2008), sendo também utilizada pelos apicultores locais para a produção de pólen monofloral.

O processo de produção do pólen apícola é singular e possui características que são determinadas de acordo com a espécie vegetal a qual a abelha forrageia. Desta forma, a classificação do pólen apícola pode ser monofloral, quando sua origem é a partir de uma única fonte botânica ou possui predomínio de um determinado tipo polínico, e multifloral, quando seu espectro apresenta uma variedade de tipos, sem que ocorra a predominância de algum específico (Modro et al., 2009; Alves; Santos, 2014).

Contudo, para essa indicação de origem monofloral ou multifloral, é importante considerar outras formas de contagem, além do padrão utilizado na melissopalinologia. A utilização de ferramentas matemáticas - como o tamanho das unidades polínicas e o volume presentes nas amostras de pólen apícola - passa a ser essencial para que não haja uma sub ou superrepresentatividade de determinado tipo polínico (Tasei et al., 1973; Silveira, 1991; Modro et al., 2009; Vossler et al., 2010; Vossler, 2015).

Nesse contexto, este estudo tem como objetivo analisar a contribuição dos grãos de pólen de Attalea funifera na produção de pólen apícola e identificar a origem botânica (taxonômica) das amostras de pólen com base no volume polínico.

\section{Material e Métodos}

\section{Área de estudo}

O município de Nilo Peçanha (13³6'13"S e 3906'14"W) está localizado no litoral do baixo sul da Bahia, sendo delimitado pelos municípios de Taperoá, Ituberá e Presidente Tancredo Neves, a uma altitude de $20 \mathrm{~m}$. Sua extensão territorial é de, aproximadamente 399,329 $\mathrm{Km}^{2}$ (Figura 1). O bioma predominante é a floresta de mata atlântica com cobertura vegetal, sendo esta associada às plantações de palmeiras, principalmente dendê e piaçava que representam a economia local.

\section{Coleta das amostras}

As amostras de pólen apícola foram obtidas diretamente com apicultores em uma região com extensas plantações de piaçava $(A$. funifera) durante o período de maio a novembro de 2014. A indicação sobre a classificação das amostras em monofloral ou multifloral foi obtida diretamente com os apicultores no momento da coleta,

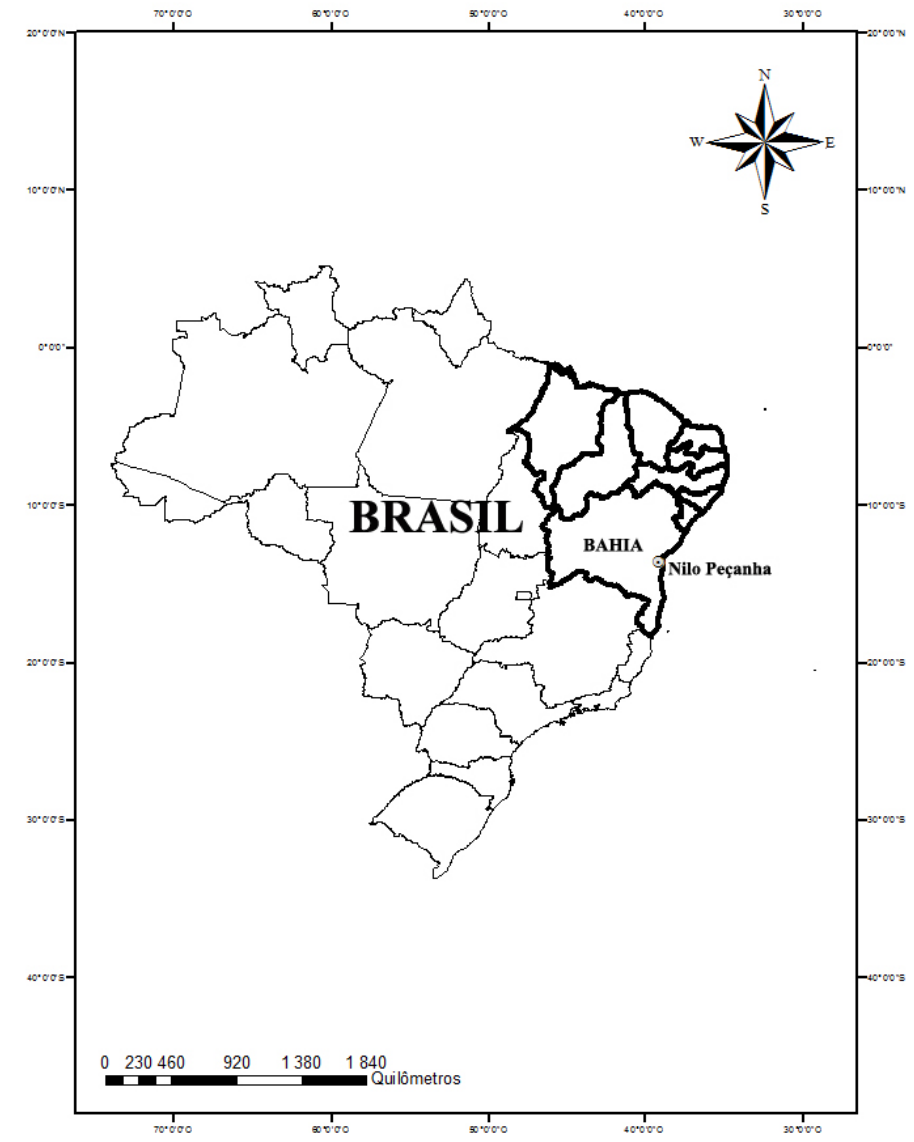

Figura 1. Local de coleta das amostras de pólen apícola no município de Nilo Peçanha, estado da Bahia, Brasil.

com base somente na coloração homogênea das amostras em associação com a florada do período.

Foram utilizadas amostras de pólen apícola desidratado (100 g) já em processamento para pré-venda ao consumidor. As amostras foram identificadas com os dados de produção e a indicação de sua origem botânica, de acordo com o produtor - todas em geral eram consideradas como "pólen monofloral de piaçava". As amostras foram acondicionadas em geladeira no Laboratório de Micromorfologia Vegetal (LAMIV) da Universidade Estadual de Feira de Santana (UEFS).

\section{Análises palinológicas}

A análise do pólen apícola foi desenvolvida conforme o protocolo de Alvarado e Delgado (1985), seguindo as modificações propostas por Novais et al. (2009). O material resultante foi acetolisado de acordo com a técnica descrita por Erdtman (1960). De cada amostra acetolisada, foram montadas cinco lâminas permanentes com gelatina glicerinada e seladas com parafina.

Uma observação geral das lâminas foi realizada para identificação e análise qualitativa dos principais tipos polínicos. Em seguida, foi feita uma análise quantitativa, com uma contagem de 500 grãos de pólen, no mínimo, por amostra, para estabelecer uma frequência de ocorrência de cada tipo polínico (Vergeron, 1964; Bucher et al., 2004). Para a classificação de frequência, foi empregada a classificação de Jones e Bryant Jr. (1996) que classifica os tipos polínicos 
presentes nas amostras nas classes muito frequente $(>50 \%)$, frequente $(20-50 \%)$, pouco frequente $(10-20 \%)$ ou raro $(<10 \%)$.

A identificação botânica dos grãos de pólen foi realizada como recomendado por Santos (2011), por comparação com a palinoteca, referência presente no LAMIV/UEFS, e com o auxílio de catálogos polínicos Moncada e Salas (1983), Roubik e Moreno (1991), Martinez-Hernandez et al. (1993), Melhem et al. (2003) e Lima et al. (2008), para o gênero Mimosa, e Bauermann et al. (2013).

\section{Análises volumétricas}

A classificação das amostras também foi realizada com base no volume dos grãos de pólen, sendo utilizados os tipos polínicos mais representativos em comparação com o tipo polínico $A$. funifera. Os tipos polínicos foram mensurados com base na metodologia de Salgado-Labouriau (1973) e, a partir dessas medidas, realizou-se o cálculo da proporção de tamanho entre os tipos polínicos selecionados.

Com base no trabalho de Vossler (2015), foi realizada uma analogia das formas dos tipos polínicos acetolizados com figuras geométricas, na qual eram feitas medidas do eixo polar e eixo equatorial. Para os tipos polínicos semelhantes às formas esféricas e elipsoides, somente a vista polar foi utilizada e para formas prismáticas as duas vistas foram usadas (vista polar e equatorial).

A categoria de tamanho aplicada para classificar os tipos polínicos foi baseada nos trabalhos de Hesse et al. (2009), no qual o tipo polínico pode ser muito pequeno $(<10 \mu \mathrm{m})$, pequeno $(10-25$ $\mu \mathrm{m})$, médio $(26-50 \mu \mathrm{m})$, grande $(51-100 \mu \mathrm{m})$ ou muito grande $(>100 \mu \mathrm{m})$. A partir dessa categorização de tamanho foram selecionados três tipos polínicos com maiores frequências nas amostras e comparados com o tipo A. funifera para saber a real contribuição de cada tipo polínico (Silveira et al., 1991; Modro et al., 2009).

\section{Resultados}

\section{Riqueza polínica}

Com base na análise qualiquantitativa dos dados verificou-se a presença de 23 tipos polínicos, pertencentes a 13 famílias (Figura 2). Dentre as famílias que mais contribuíram para esse espectro, destaque para Fabaceae (6), Euphorbiaceae (5) e Asteraceae (2) que juntas representaram mais de $46 \%$ do total de tipos polínicos identificados. As demais famílias (10) apresentaram somente um tipo polínico.

Dentre as amostras analisadas de pólen apícola com indicação monofloral, aproximadamente $58 \%$ das amostras tiveram todos os tipos polínicos identificados. Contudo, mesmo com a existência de tipos indeterminados, isso não comprometeu a avaliação dos resultados, pois a quantidade destes nas amostras foi baixa $(<3 \%)$.

Tabela 1. Frequência dos tipos polínicos encontrados nas amostras de pólen apícola produzido no município de Nilo Peçanha, Bahia, Brasil.

\begin{tabular}{|c|c|c|c|c|c|c|c|c|}
\hline \multirow{2}{*}{$\begin{array}{l}\text { Família/Tipos } \\
\text { polínicos }\end{array}$} & \multirow[b]{2}{*}{ F.O. } & \multicolumn{7}{|c|}{ Meses } \\
\hline & & Maio & Jun & Jul & Ago & Set & Out & Nov \\
\hline \multicolumn{9}{|l|}{ Anacardiaceae } \\
\hline Spondias & PF & & & & & & & 0,4 \\
\hline \multicolumn{9}{|l|}{ Aquifoliaceae } \\
\hline Ilex & PF & & & & & & & 8,2 \\
\hline \multicolumn{9}{|l|}{ Arecaceae } \\
\hline Attalea funifera & MF & 1,6 & 0,2 & 0,6 & 4,6 & 2,0 & 4,6 & 62,7 \\
\hline \multicolumn{9}{|l|}{ Asteraceae } \\
\hline Mikania & MF & 7,2 & & 0,6 & 4,8 & 1,4 & 2,0 & 1,2 \\
\hline Vernonantbura & $\mathrm{F}$ & & & & 0,4 & & & 0,2 \\
\hline \multicolumn{9}{|l|}{ Celastraceae } \\
\hline Maytenus & F & & 4,1 & & & & & 0,6 \\
\hline \multicolumn{9}{|l|}{ Cyperaceae } \\
\hline Rhynchospora & MF & 5,0 & 1,0 & 3,2 & 0,6 & 1,4 & 1,0 & 4,8 \\
\hline \multicolumn{9}{|l|}{ Euphorbiaceae } \\
\hline Alchornea & $\mathrm{F}$ & & & & & 0,2 & & 6,2 \\
\hline Euphorbiaceae 1 & PF & & & & & & 11,1 & \\
\hline Euphorbiaceae 2 & PF & & & & & & 28,0 & \\
\hline Euphorbiaceae 3 & PF & & & & & & 1,8 & \\
\hline Ricinus & PF & & & & & & & 0,2 \\
\hline \multicolumn{9}{|l|}{ Fabaceae } \\
\hline Fabaceae 1 & $\mathrm{PF}$ & & & & & & & 0,4 \\
\hline Fabaceae 2 & PF & & & & & & 0,6 & \\
\hline Fabaceae 3 & $\mathrm{~F}$ & & 0,2 & & & & & 0,2 \\
\hline Mimosa acutistipula & $\mathrm{F}$ & & 0,4 & 0,2 & & 1,0 & & \\
\hline Mimosa pudica & MF & 85,5 & 94,1 & 95,2 & 88,7 & 93,1 & 42,1 & 5,4 \\
\hline Stryphnodendron & $\mathrm{PF}$ & 0,4 & & & & & & \\
\hline \multicolumn{9}{|l|}{ Malpighiaceae } \\
\hline Malpighiaceae 1 & $\mathrm{PF}$ & & & & & 0,2 & & \\
\hline \multicolumn{9}{|l|}{ Myrtaceae } \\
\hline Myrcia & MF & & & 0,2 & 0,8 & & 4,2 & 0,2 \\
\hline \multicolumn{9}{|l|}{ Loranthaceae } \\
\hline Loranthaceae 1 & $\mathrm{PF}$ & & & & & & & 1 \\
\hline \multicolumn{9}{|l|}{ Tiliaceae } \\
\hline Tiliaceae 1 & PF & 0,4 & & & & & & \\
\hline \multicolumn{9}{|l|}{ Urticaceae } \\
\hline Cecropia & F & & & & & & & \\
\hline Indeterminados & & & & & & $\begin{array}{l}0,8 \\
(1)\end{array}$ & $\begin{array}{l}0,4 \\
(2)\end{array}$ & $\begin{array}{l}2,8 \\
(2)\end{array}$ \\
\hline Total (\%) & & 100 & 100 & 100 & 100 & 100 & 100 & 100 \\
\hline
\end{tabular}

Em relação à riqueza polínica mensal nas amostras, notou-se que o mês de novembro apresentou a maior riqueza (19 tipos polínicos), seguido pelos meses de setembro e outubro (12 e 8 , respectivamente) (Tabela 1). Já os meses de maio a julho exibiram a menor riqueza polínica (6). Além disso, foi percebido também que apenas os tipos polínicos A. funifera (Arecaceae), M. pudica (Fabaceae) e Rhynchospora (Cyperaceae) estiveram presentes em todas as amostras. Dentre as amostras analisadas, cinco tipos polínicos se apresentaram como muito frequente (A. funifera, Mikania, M. pudica, Myrcia e Rhynchospora), enquanto que os demais tipos foram classificados como frequentes (6) ou pouco frequentes (12).

Tabela 2. Origem botânica, forma e contribuição dos principais tipos polínicos em $100 \mathrm{~mL}$ de pólen apícola, volume por unidade polínica x frequência.

\begin{tabular}{|c|c|c|c|c|c|}
\hline Tipo polínico & Forma & $\begin{array}{l}\text { Volume real da } \\
\text { unidade polínica } \\
\qquad\left(\mu \mathrm{m}^{3}\right)\end{array}$ & $\begin{array}{c}\text { Frequência } \\
\%\end{array}$ & $\begin{array}{l}\text { Volume estimado no } \\
\text { pólen apícola } \\
\text { com base no } \% \\
\left(\mu \mathrm{m}^{3}\right)\end{array}$ & $\begin{array}{l}\text { Volume estimado no pólen } \\
\text { apícola com base no volu- } \\
\text { me (mL) }\end{array}$ \\
\hline Attalea funifera & Prisma & $93.974,70$ & 4,6 & $2.161 .418,10$ & 88,71 \\
\hline Mikania & Esfera & $8.059,89$ & 4,8 & $193.437,36$ & 8,22 \\
\hline Mimosa pudica & Esfera & 523,33 & 88,7 & $230.788,53$ & 0,27 \\
\hline Rhynchospora & Cone & $21.971,64$ & 0,6 & $65.914,92$ & 2,80 \\
\hline
\end{tabular}



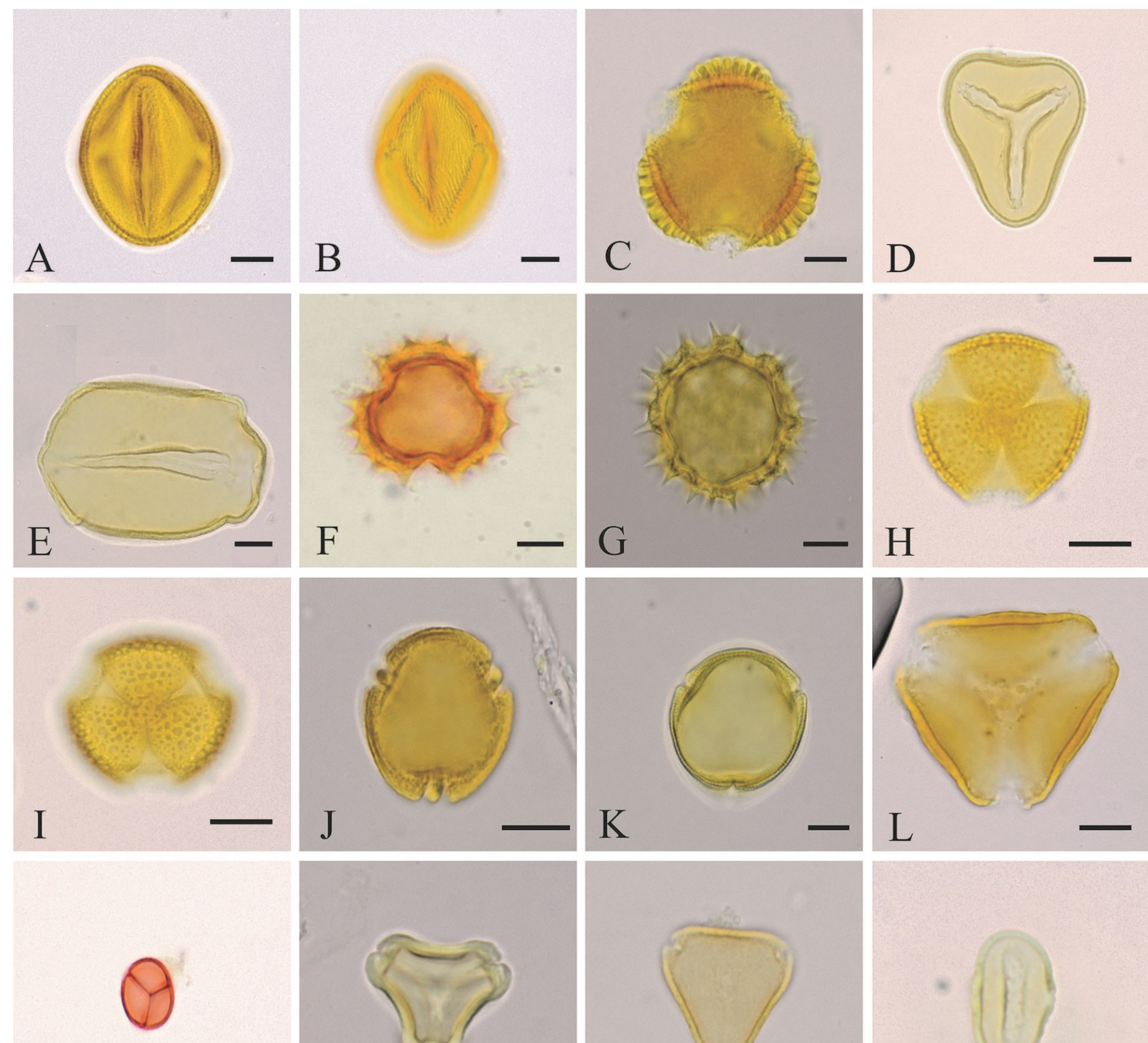

\section{M}
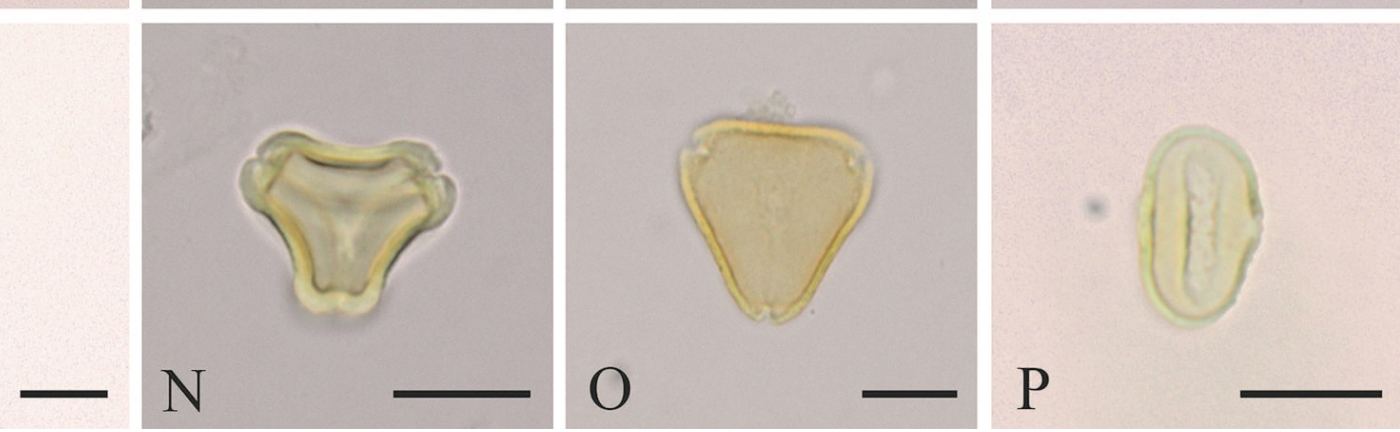

Figura 2. Tipos polínicos encontrados nas amostras de pólen apícola monofloral de Arecaceae no município de Nilo Peçanha, Bahia, Brasil. A-B. Anacardiaceae: Spondias. C. Aquifoliaceae: Ilex. D-E. Arecaceae: Attalea funifera. F-G. Asteraceae: Mikania (F); Vernonanthura (G). H-I. Celastraceae: Maytenus. J-K. Euphorbiaceae: Alchornea (J); Ricinus (K). L. Loranthaceae: Loranthaceae 1. M. Fabaceae: Mimosa pudica (M). N-O. Myrtaceae: Eucalyptus (N); Myrcia (O). P. Urticaceae: Cecropia. (Escala: $10 \mu \mathrm{m})$.

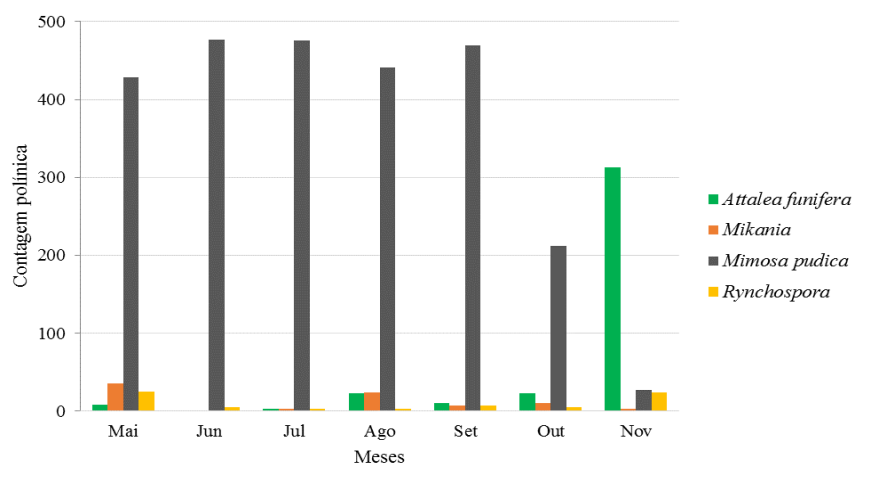

Figura 3. Ocorrência e quantidade dos principais tipos polínicos presentes nas amostras de pólen apícola com base no censo polínico.

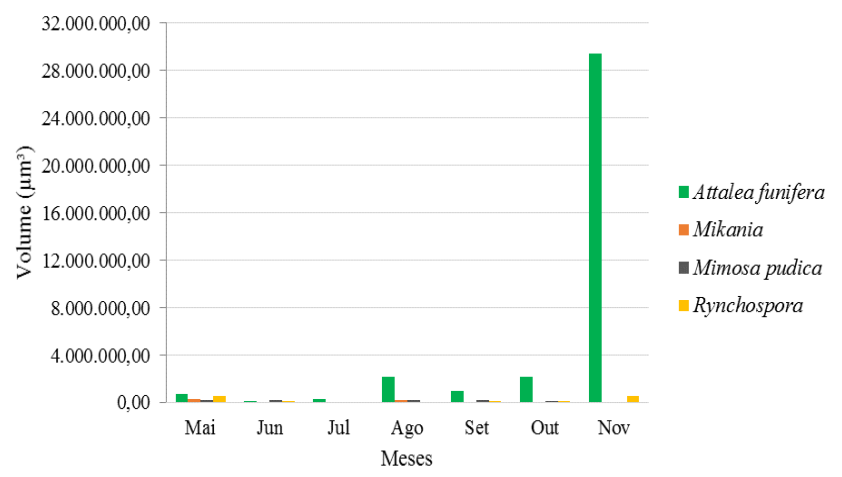

Figura 4. Análise volumétrica dos principais tipos polínicos presentes nas amostras de pólen apícola. 


\section{Análise do volume polínico}

Os tipos polínicos com maiores frequências nas amostras tiveram calculados o respectivo volume de sua unidade polínica, a fim de se obter uma estimativa da contribuição individual de cada tipo polínico nas amostras (Tabela 2). A análise volumétrica mostrou um resultado diferente daquele encontrado na análise da frequência polínica. Somente a amostra de junho/2014 não teve o tipo A. funifera como predominante na amostra. $\mathrm{Na}$ contagem polínica houve uma superrepresentatividade do tipo Mimosa pudica (Figura 3), enquanto que na estimativa de volume o destaque foi $A$. funifera (Figura 4).

Essa análise volumétrica também contribuiu para categorizar as amostras em monoflorais ou multiflorais, com base na contribuição dos respectivos tipos polínicos nas amostras. Assim, dentre as sete amostras analisadas, seis foram consideradas monoflorais de A. funifera com base no volume.

\section{Discussão}

As informações obtidas com os apicultores sobre as amostras de pólen apícola não foram confirmadas após a análise padrão (frequência polínica), ou seja, a indicação de que as amostras eram compostas por pólen apícola monofloral de piaçava não foi confirmada com base no censo polínico (Tabela 2). Uma explicação está na presença expressiva do tipo polínico de Mimosa pudica, sendo incomparável, no que se refere à ocorrência, a qualquer outro tipo polínico dentre os identificados nas amostras. Isto pode ser explicado pelo 'boom' de floração, característico da família Fabaceae. Além disso, a acessibilidade das abelhas às flores das respectivas plantas, que são altamente poliníferas, facilita o aparecimento em larga escala nos produtos apícolas. Segundo Arroyo (1981), as abelhas e a família Fabaceae estão intimamente associadas entre si pela história evolutiva e esse sucesso evolutivo está relacionado diretamente com a polinização.

O tipo polínico A. funifera, por sua vez, esteve presente em todas as amostras, fato este explicado pela proximidade do apiário às culturas de piaçava, mas, sua frequência dentro das amostras, de acordo com a contagem polínica, foi muito baixa (Tabela 2, Figura 3). Contudo, em uma análise baseada no volume, percebe-se uma inversão de contribuição polínica e, por conseguinte, de classificação das amostras. Essa situação ocorre simplesmente porque o volume do tipo polínico $A$. funifera apresentou maior contribuição quando comparado ao tipo M. pudica (Figura 4). Esse cenário já foi encontrado em outros trabalhos que tentaram relacionar a frequência dos tipos polínicos ao respectivo volume (Tasei, 1973; Silveira, 1991; Biesmeijer et al., 1992; Vossler et al., 2010; Vossler, 2015; Alves e Santos, 2017).

O tipo polínico $A$. funifera apresenta um tamanho considerado grande $(99,95 \mu \mathrm{m})$ quando comparado aos demais tipos presentes com maiores frequências como, por exemplo, M. pudica $(10,0 \mu \mathrm{m})$, Mikania $(25,0 \mu \mathrm{m})$ e Rhynchospora $(33,5 \mu \mathrm{m})$; sua contribuição volumétrica é significativa, mesmo em baixas concentrações nas amostras (Tabela 1). Assim, apenas a contagem polínica não é um parâmetro suficiente para diagnosticar uma amostra em monofloral ou multifloral; é necessário considerar o tamanho e o volume dos tipos polínicos em adição à sua frequência (Alves e Santos, 2017).
Outro ponto a ser discutido nesse estudo é a presença de tipos polínicos anemófilos como, por exemplo, Rhynchospora. Apesar de apresentar essa síndrome da polinização, a frequência desse tipo foi considerada alta em algumas amostras. Logo, pode-se inferir que esse tipo polínico foi coletado pelas abelhas e não apenas foi transportado até a colmeia pelo vento. Segundo Dahlgren et al. (1985), a anemofilia é a estratégia mais comum na família Cyperaceae, sendo rara a entomofilia. Contudo, Ramos e Fonseca (2006) afirmam que alguns representantes da família apresentam ambifilia, isto é, síndrome de polinização entomófila e anemófila.

\section{Conclusão}

Apesar das amostras indicarem uma origem botânica, destacando a alta frequência do pólen de $M$. pudica, a análise volumétrica indicou o inverso, com o tipo polínico A. funifera apresentando elevado potencial na composição do pólen apícola. O fato está diretamente atrelado ao tamanho dos grãos de pólen, cujo mais frequente (M. pudica) é classificado como pequeno e apresenta-se em grande quantidade para coleta. Em contrapartida, o pólen de $A$. funifera, considerado um pólen grande, apresenta um maior volume e, consequentemente, é apresentado em menor quantidade para a coleta.

A utilização de outros parâmetros, além da contagem polínica, passa a ser uma nova ferramenta que ainda precisa ser aprimorada, pois a diversidade de formas e tamanhos polínicos dificulta a aplicação dessas análises, especialmente em área de grande biodiversidade vegetal, como o Brasil. Por outro lado, esses dados evidenciam a importância de análises volumétricas, não somente para a determinação da origem botânica, como também para a determinação da importância nutricional do pólen apícola.

\section{Agradecimentos}

Os autores agradecem à CAPES pela bolsa de doutorado para RFA e ao CNPq pela bolsa científica para FARS. Aos apicultores do município de Nilo Peçanha/Bahia pela doação de amostras de pólen utilizadas neste estudo, ao Programa de Pós-Graduação em Botânica e ao Laboratório de Micromorfologia Vegetal da Universidade Estadual de Feira de Santana por permitir o uso de suas instalações para esta pesquisa (LAMIV-UEFS).

\section{Referências}

Alvarado JL, Delgado MD. Flora apícola en Uxpanapa, Veracruz, Mexico. Biotica 1985;10:257-275.

Alves RF, Santos FAR. Arecaceae potential for production of monofloral bee pollen. Grana 2017;56:1-10.

Arroyo MTK. Breeding systems and pollination biology in Leguminosae. In: Polhil RM, Raven PH (eds.). Advances in legumes systematics. Kew: Royal Botanic Gardens; 1981.

Avelar FF. Utilização de fibras de piaçava (Attalea funifera) na preparação de carvão ativado. Dissertação [Mestrado em Agroquímica] - Universidade Federal de Lavras; 2008.

Barreto LMRC, Funari SRC, Orsi RO. Composição e qualidade do pólen apícola proveniente de sete Estados brasileiros e do Distrito Federal. Boletim Indústria Animal, 2005;62(2):167-175. 
Bauermann SG, Radaeski JN, Evaldt ACP, Queiroz EP, Mourelle D, Prieto AR, Silva CI. Pólen nas angiospermas: diversidade e evolução. Canoas, Editora Ulbra; 2013.

Biesmeijer JC, van Marwiik B, van Deursen K, Punt W, Sommeijer MJ. Pollen sources for Apis mellifera L. (Hym. Apidae) in Surinam, based on pollen grain volume estimates. Apidologie 1992;23:245-256.

Brasil, Ministério de Agricultura e do Abastecimento [Internet]. Instrução normativa n. ${ }^{\circ}$ 3, de 19 de janeiro de 2001. Regulamento técnico de identidade e qualidade do pólen apícola. Diário Oficial [da] República Federativa do Brasil. 2001 jan. 23; Seção 16-I. p. 18-23. [acesso em 11 abr 2016]. Disponível em: http:// www.prodapys.com.br/navega.php? idioma $=$ fr\&item $=$ qualidade\&sub $=\mathrm{aV}$.

Bucher E, Kofler V, Vorwohl G, Zieger E. Lo spettro pollinico dei mieli dell'Alto Adige. Laives: Agenzia Provinciale per la Protezione dell'Ambiente e la Tutela del Lavoro; 2004.

Dahlgren R, Clifford HT, Yeo PF. The families of the monocotyledons: structure, evolution and taxonomy. Berlin; New York: Springer-Verlag; 1985.

Erdtman G. The acetolysis method. A revised description. Svensk Botanisk Tidskrift 1960;54:561-564.

Hesse M, Halbritter H, Weber M, Zetter R, Frosch-Radivo A, Buchner R. Pollen terminology: an illustrated handbook. New York: Springer-Verlag; 2009.

Jones GD, Bryant VM. Melissopalynology. In: Jansonius J, McGregor DC (eds.). Palynology: principles and applications. St. Louis: AASP Foundation; 1996. p. 933-938.

Lima LCL, Silva FHM, Santos FAR. Palinologia de espécies de Mimosa L. (Leguminosae-Mimosoideae) do Semiárido brasileiro. Acta Botanica Brasilica 2008; 22(3): 794-805.

Lorenzi H, Noblick LR, Kahn F, Ferreira E. Flora brasileira: Arecaceae (Palmeiras). Nova Odessa: Instituto Plantarum; 2010.

Martínez-Hernandéz, E, Cuadriello-Aguilar JI, Téllez-Valdez O, Ramírez-Arriaga E, Sosa-Nájera MS, Melchor-Sánchez JEM, Medina-Camamcho M, Lozano-García MS. Atlas de las plantas y el polen utilizados por las cinco especies principales de abejas productoras de miel en la Region del Tacana, Chiapas, México. México: Instituto de Geología, Universidad Nacional Autonoma de México; 1993.

Melhem TS, Cruz-Barros MAV, Corrêa MAS, Makino-Watanabe H, Silvestre-Capelato MSF, Esteves VLG. Variabilidade polínica em plantas de Campos do Jordão (São Paulo, Brasil). Boletim do Instituto de Botânica 2003;16:1-104.

Modro AFH, Maia E, Silva IC, Luz CFP, Message D. Subamostragem de pólen apícola para análise melissopalinológica. Hoehnea 2009;36(4):709-714.

Moncada M, Salas E. Pólen de las plantas melíferas en Cuba. Havana: Centro de Información y Divulgación Agropecuário; 1983.

Novais JS, Lima LCL, Santos FAR. Botanical affinity of pollen harvested by Apis mellifera L. in a semi-arid area from Bahia, Brazil. Grana 2009;48:224-234.

Ramos COC, Fonseca RBS. Polinização de Rhynchospora almensis D. A. Sympson (Cyperaceae), no município de Palmeiras, Chapada Diamantina, Bahia, Brasil. Biologia e ecologia da polinização: curso de campo/ Instituto de Biologia da UFBA, Programa de Pós-Graduação em Ecologia e Biomonitoramento. Salvador: EDUFBA; 2006.

Roubik DW, Moreno PJE. Pollen and spores of Barro Colorado island. St. Louis: Missouri Botanical Garden; 1991.

Salgado-Labouriau ML. Contribuição à palinologia dos cerrados. Academia Brasileira de Ciências, Rio de Janeiro; 1973

Santos FAR. Identificação botânica do pólen apícola. Magistra 2011;23:4-9.

Silveira FA. Influence of pollen grain volume on the estimation of the relative importance of its source to bees. Apidologie 1991;22:495-502.
Tasei JN. Comportement de nidification chez Osmia (Osmia) cornuta Latr et Osmia (Osmia) rufa L. (Hymenoptera, Megachilidae). Apidologie 1973;4:195-225.

Vergeron P. Interprétation statistique des résultats en matière d'analyse pollinique des miels. Annl Abeille 1964; 7(4):349-364.

Vossler FG, Tellería MC, Cunningham M. Floral resources foraged by Geotrigona argentina (Apidae, Meliponini) in the Argentine Dry Chaco forest. Grana 2010; 49: 142-153.

Vossler FG. Small pollen grain volumes and sizes dominate the diet composition of three South American subtropical stingless bees. Grana; 2015; 54(1): 68-81.

\section{Análisis volumétrico del polen de Attalea funi- fera Mart. (Arecaceae) en muestras de polen de abeja en la costa del bajo sur da Bahía, Brasil}

Se realizó el análisis volumétrico del polen de Attalea funifera Mart. (Arecaceae) en muestras de polen apicola comercializado en el municipio de Nilo Peçanha, Babia, en el periodo de mayo a noviembre de 2014. Las muestras fueron identificadas y cuantificadas, a fin de determinar el origen botánico y la la importancia de A. funifera en la composición del polen apicola, bajo el aspecto volumétrico. El espectro polínico fue compuesto por 23 tipos polinicos distribuidos en 13 familias botánicas, teniendo como las más representativas las familias Fabaceae, Euphorbiaceae y Asteraceae. El tipo polinico más frecuente fue Mimosa pudica, pero los análisis volumétricos apuntaron a A. funifera como el más importante en la constitución del polen recogido. Por lo tanto, con base en el volumen $\left(93.974,70 \mu^{3}\right)$ se verifica el potencial del polen de $\underline{A}$. funifera en muestras del polen apicola y consecuentemente la importancia de análisis volumétricos además de los análisis cuantitativos y de frecuencia.

Palabras clave: Apicultura. Pólen apicola. Palinología. Volumen polínico. Apis mellifera.

\section{Analyse volumétrique du pollen Attalea funifera Mart. (Arecaceae) dans des échantillons de pollen d'abeille sur la côte sud de Bahia, au Brésil}

L'analyse volumétrique du pollen d'Attalea funifera Mart a été réalisée. (Arecaceae) dans des échantillons de pollen d'abeille commercialisés à Nilo Peçanha, Babia, de mai à novembre 2014. Les échantillons ont été identifiés et quantifiés dans le but de déterminer l'origine botanique et l'importance de l'A. funifera dans la composition du pollen d'abeille, sous l'aspect volumétrique. Le spectre pollinique était composé de 23 types de pollen répartis dans 13 familles botaniques, les familles les plus représentatives étant les Fabaceae, les Euphorbiaceae et les Asteraceae. Le type de pollen le plus fréquent était 
Mimosa pudica, mais les analyses volumétriques ont indiqué que l'A. funifera était le plus important dans la constitution du pollen collecté. Sur la base du volume (93.974,70 um $^{3}$ ), on constate donc le potentiel de pollen $d^{\prime}$ A. funifera dans des échantillons de pollen d'abeille et par conséquent, l'importance de l'analyse volumétrique en addition aux analyses quantitatives et fréquentielles.

Mots clés: Apiculture. Pollen d'abeille. Palynologie. Volume pollinique. Apis mellifera.

Este artigo possui erratum disponível em: https://doi.org/10.33447/paubrasilia.2021.e0073er

This article has erratum available at: https://doi.org/10.33447/paubrasilia.2021.e0073er

Este artículo tiene erratum publicada en: https://doi.org/10.33447/paubrasilia.2021.e0073er

\section{ERRATUM}

No artigo "Análise volumétrica do pólen de Attalea funifera Mart. (Arecaceae) em amostras de pólen apícola do litoral do baixo sul da Bahia, Brasil”, com número de doi: 10.33447/paubrasilia.v1i2.14, publicado na revista Paubrasilia, 1(2):5-11, na página 7. Correção em referência citada no segundo parágrafo da subseção "Análises volumétricas".

onde se lia:

Vossler (2014)

leia-se: 